\title{
Long Palindromic Sequences Induce Double-Strand Breaks during Meiosis in Yeast
}

\author{
FAROOQ NASAR, ${ }^{1}$ CRAIG JANKOWSKI, ${ }^{1}$ AND DILIP K. NAG ${ }^{1,2 *}$ \\ Molecular Genetics Program, Wadsworth Center, ${ }^{1}$ and Department of Biomedical Sciences, \\ School of Public Health, State University of New York, ${ }^{2}$ Albany, New York 12201
}

Received 9 December 1999/Returned for modification 7 February 2000/Accepted 28 February 2000

\begin{abstract}
Inverted-repeated or palindromic sequences have been found to occur in both prokaryotic and eukaryotic genomes. Such repeated sequences are usually short and present at several functionally important regions in the genome. However, long palindromic sequences are rare and are a major source of genomic instability. The palindrome-mediated genomic instability is believed to be due to cruciform or hairpin formation and subsequent cleavage of this structure by structure-specific nucleases. Here we present both genetic and physical evidence that long palindromic sequences $(>50 \mathrm{bp}$ ) generate double-strand breaks (DSBs) at a high frequency during meiosis in the yeast Saccharomyces cerevisiae. The palindrome-mediated DSB formation depends on the primary sequence of the inverted repeat and the location and length of the repeated units. The DSB formation at the palindrome requires all of the gene products that are known to be responsible for DSB formation at the normal meiosis-specific sites. Since DSBs are initiators of nearly all meiotic recombination events, most of the palindrome-induced breaks appear to be repaired by homologous recombination. Our results suggest that short palindromic sequences are highly stable in vivo. In contrast, long palindromic sequences make the genome unstable by inducing DSBs and such sequences are usually removed from the genome by homologous recombination events.
\end{abstract}

Unusual DNA sequence arrangements and DNA structures are a major source of genomic instability. Inverted repeated sequences are an example of such sequence arrangements and are also known to cause different types of genomic rearrangements in a wide variety of organisms $(2,17,27,40)$. The presence of the inverted repeats is known to stimulate deletion formation and also increases both inter- and intrachromosomal recombination between homologous sequences $(9,16$, 17, 27, 31, 40, 50, 57). These palindrome-mediated genomic rearrangements depend on the length of the repeated units, as palindromic sequences shorter than $30 \mathrm{bp}$ are highly stable while long palindromic sequences are difficult to maintain in vivo $(2,9,27,31,40-42)$.

Inverted repeated sequences have the potential to form stem-loop (hairpin or cruciform) structures by intrastrand base pairing in the single-stranded DNA. Such a stem-loop structure may form during the course of normal DNA metabolism. For example, during replication, the single-stranded regions which are likely to be present on the lagging strand may facilitate hairpin formation $(47,54)$. In addition, environmental factors or changes in chromatin structure may cause alterations in the local superhelical density, leading to extrusion into cruciform conformation. The hairpin structures are potential substrates for several structure-specific nucleases and/or mismatch repair enzymes, and the action of such an enzyme may result in a double-stranded lesion in the DNA. Supporting this idea is the observation that the stability of long palindromic sequences is increased significantly in nuclease-deficient $(s b c C D)$ strains of Escherichia coli (27). A double-strand break (DSB) in DNA may lead to complete or partial loss of the genomic material or can initiate homologous recombination events $(2,27,28,29$, 31, 40, 44).

\footnotetext{
* Corresponding author. Mailing address: Molecular Genetics Program, Wadsworth Center, 120 New Scotland Ave., Albany, NY 122012002. Phone: (518) 473-6327. Fax: (518) 474-3181. E-mail: dilip.nag @wadsworth.org.
}

Cruciform formation by palindromic sequences has been demonstrated in vitro in negatively supercoiled DNA $(27,30$, $35)$. However, palindromes below the size limit that causes inviability in the wild-type host are hard to detect in the cruciform conformation. Specifically designed palindromes or specialized hosts and/or conditions are required to detect a significant amount of cruciform DNA $(6,27,33,52,60)$. Nag et al. developed a genetic method to detect hairpin structures in vivo in the yeast Saccharomyces cerevisiae (42). This method is based on trapping of the hairpin structures in the form of heteroduplex DNA, an intermediate of genetic recombination.

The heteroduplex intermediate is formed during genetic recombination by transfer of a DNA strand from a donor chromosome to a recipient chromosome, and the formation of heteroduplex DNA (hDNA) is usually monitored by following the segregation of heterozygous markers during meiosis (44, 46). In yeast, a heterozygous marker (alleles A and a) during meiosis normally follows the 4A:4a Mendelian segregation pattern (following the nomenclature of eight-spore-producing fungi). However, 6A:2a or 2A:6a gene conversion events, and postmeiotic segregation (PMS) events in which one or more spore colonies generated from a single tetrad show sectored colonies (e.g., 5A:3a, 3A:5a, and aberrant 4A:4a) for the heterozygous marker are also observed (46). If the hDNA is formed between a wild-type gene and a mutant allele, a mismatch is generated. Gene conversion events occur by the repair of mismatches in the hDNA, whereas a failure to repair the mismatch results in a PMS event (46). Consequently, a defect in any of the mismatch repair genes increases the rate of PMS events for alleles which otherwise would produce only gene conversion events. For most heterozygous alleles, PMS rates are low (3 to $19 \%$ per aberrant segregation), indicating that mismatches in the hDNA are efficiently repaired by the mismatch repair system in yeast $(4,11,14)$. The base substitution mutations that led to a C-C mismatch in the hDNA and palindromic-insertion mutations show high rates of PMS 
events ( 35 to 54 and 60 to $80 \%$ per aberrant segregation, respectively) $(4,11,14,40,42)$.

A heteroduplex formed involving a wild-type strand and a palindromic-insertion mutant strand would contain a hairpin structure because of the presence of the complementary sequence in the inserted sequence on the mutant strand. These hairpin structures are poorly repaired by the mismatch repair system in yeast (40-42). As a result, palindromic-insertion mutant alleles exhibit a high rate of PMS events. The palindromic insertions in these studies were short (14 to $36 \mathrm{bp}$ ) (40-42). Recently, a 140-bp long palindromic-insertion mutant allele has been shown to decrease the rate of PMS events with a simultaneous increase in the rate of gene conversion events. In addition, among the conversion events, there is a strong disparity between the $6: 2$ and 2:6 events (40). These results indicate that the 140-bp palindromic-insertion mutant allele is likely to act as a meiotic recombination hotspot, i.e., a recombination initiation site.

Most meiotic recombination is initiated by a $\operatorname{DSB}(29,44)$, and physical analysis has confirmed that the presence of the 140-bp palindrome induces DSBs during meiosis (40). It has been proposed that long palindromic sequences undergo cruciform formation at a higher frequency than short palindromes and the resulting cruciform structure is resolved by structurespecific nucleases, leading to DSB formation. Since only one long palindrome has been tested, there remains a possibility that the primary sequence rather than the structure of the inserted inverted repeat is responsible for inducing DSBs. In this report, we show that it is the length of the palindromic insertion rather than the primary sequence that is responsible for inducing DSBs during meiosis. However, the primary sequence of the inverted repeat also plays a significant role in determining the strength of the recombination initiation site. Palindrome-mediated DSB formation requires all of the gene products that are known to be necessary for normal meiosisspecific DSB formation.

\section{MATERIALS AND METHODS}

Yeast strains. The genotypes of all of the relevant yeast strains used in this study are shown in Table 1 . All of the yeast strains used in this study were derived from AS4 and AS13. The HIS4 locus in the AS13 $\times$ AS4 background shows a high rate of meiotic recombination (42). The his 4 mutant alleles containing different palindromic insertions were introduced into the chromosome by replacing the wild-type gene in a two-step gene replacement procedure (49). The resulting His ${ }^{-}$haploids were confirmed by Southern hybridization. The rad50S mutation was introduced by a one-step gene replacement method as described before (40). All genetic manipulations were carried out by standard procedures (48). The tester strains for the allelism test were constructed as follows. DBY939 ( $\alpha$ suc2-215 ade2-101) was mated with FNY7 (AS13 his4-140a), and the diploids were sporulated. From the dissected spores, DNY285 ( $\alpha$ ade2 his4-140a) and DNY286 (a ade2 his4-140a) were selected. Similarly, DBY939 was mated with DNY63 (AS13 his4-140) to generate the DNY287 ( $\alpha$ ade2 his4-140) and DNY288 (a ade2 his4-140) haploid tester strains.

Media. The presporulation medium contained $0.5 \%$ yeast extract, $1 \%$ peptone, $0.67 \%$ yeast nitrogen base without amino acids, $1 \%$ potassium acetate, and $0.05 \mathrm{M}$ potassium biphthalate, and the $\mathrm{pH}$ was adjusted to 5.5. The sporulation medium contained $1 \%$ potassium acetate with one-fifth of the nutritional requirements. All of the other media used were as described by Rose et al. (48).

Plasmids. Standard molecular biology procedures were used for all plasmid constructions (32). All oligonucleotides were inserted into the SalI site of the plasmid pDN9 (42). The sequences of the inserted oligonucleotides are shown in Table 2. The plasmid pDN9 was constructed by inserting an XhoI-BglII fragment of the HIS4 coding sequence into BamHI-SalI-treated YIp5. This construction leaves a unique SalI site within the HIS4 sequence. The his4 mutant alleles containing inverted repeats of different lengths were constructed by sequential addition of palindromic oligonucleotides into the SalI site of pDN9. The first plasmid, pDN19, has a 32-bp palindromic insertion (his4-IR16) (41) at the SalI site of pDN9. The inserted inverted repeat has a BamHI site at its center of symmetry. The plasmid pDN19 was digested with BamHI, and a 38-bp palindrome with a SalI site at its center of symmetry was ligated into the BamHI site. The resulting plasmid, pDN73, has a 70-bp palindromic sequence inserted at the SalI site of the HIS4 sequence.
TABLE 1. Yeast strains used in this study

\begin{tabular}{|c|c|}
\hline Strain & Genotype \\
\hline \multicolumn{2}{|c|}{ AS4 ............................. } \\
\hline \multicolumn{2}{|c|}{ AS13............................MATa leu2-Bst ade6 ura3 } \\
\hline \multicolumn{2}{|c|}{ DNY64........................AS4 × AS13 with his4-140 } \\
\hline \multicolumn{2}{|c|}{ DNY214 ....................... NY64 but homozygous for rad50s } \\
\hline \multicolumn{2}{|c|}{ DNY237....................AS13 with his4-140 spo13-D4 } \\
\hline \multicolumn{2}{|c|}{ DNY238.....................AS4 with his4-713 leu2-RI spo13-D4 } \\
\hline \multicolumn{2}{|c|}{ CJY1 ............................. } \\
\hline \multicolumn{2}{|c|}{ CJY2 …......................... JY1 but homozygous for mei4 } \\
\hline \multicolumn{2}{|c|}{ CJY11 ........................... JY1 but homozygous for rad50 } \\
\hline \multicolumn{2}{|c|}{ CJY15 ….......................YY1 but homozygous for rec102 } \\
\hline \multicolumn{2}{|c|}{ CJY19 ......................... JY1 but homozygous for rec104 } \\
\hline \multicolumn{2}{|c|}{ CJY24 „.........................YY1 but homozygous for mre11 } \\
\hline \multicolumn{2}{|c|}{ CJY25 ........................... JY1 but homozygous for xrs2 } \\
\hline \multicolumn{2}{|c|}{ CJY29 ...........................YY1 but homozygous for spo11 } \\
\hline \multicolumn{2}{|c|}{ CJY30 .......................... JY1 but homozygous for rec114 } \\
\hline \multicolumn{2}{|c|}{ CJY57 ...........................YY1 but homozygous for mer2 } \\
\hline \multicolumn{2}{|c|}{ CJY38 ........................... JY29 but homozygous for rad50S } \\
\hline \multicolumn{2}{|c|}{ DNY282......................... but homozygous for rad50S } \\
\hline \multicolumn{2}{|c|}{ FNY2 ….....................AS4 × AS13 with his4-105 } \\
\hline \multicolumn{2}{|c|}{ FNY4 ........................AS4 $\times$ AS13 with his4-140a } \\
\hline \multicolumn{2}{|c|}{ FNY6 ..........................AS4 × AS13 with his4-108 } \\
\hline \multicolumn{2}{|c|}{ FNY10 .....................AS4 $\times$ AS13 with his $4-120$} \\
\hline \multicolumn{2}{|c|}{ FNY16 .......................AS4 × AS13 with his4-70 } \\
\hline \multicolumn{2}{|c|}{ FNY18 ....................... 4 AS13 with his4-116 } \\
\hline \multicolumn{2}{|c|}{ DNY225 .....................AS4 × AS13 with his4-100 } \\
\hline \multicolumn{2}{|c|}{ DNY115 .....................AS4 $\times$ AS13 but homozygous for rad50S } \\
\hline \multicolumn{2}{|c|}{ FNY8 …........................ } \\
\hline \multicolumn{2}{|c|}{ FNY13 ........................... 6 but homozygous for rad50S } \\
\hline FNY14 .. & ...FNY10 but homozygous for rad50S \\
\hline FNY23.. & ...DNY225 but homozygous for rad50S \\
\hline FNY24.. & ...FNY16 but homozygous for rad50S \\
\hline FNY26.. & ...FNY4 but homozygous for msh2 \\
\hline DNY270 & ..FNY10 but homozygous for $m s h 2$ \\
\hline FNY31 .. & ..FNY4 but homozygous for rad1 \\
\hline DNY274 & ...AS4 with rad50s $\times$ AS13 with his4-IR16 rad50s \\
\hline FNY34 .. & ...DNY64 but homozygous for his4- $\Delta 52$ \\
\hline FNY37 .. & ..FNY4 but homozygous for his4- $\Delta 52$ \\
\hline FNY39 .. & ...FNY37 but homozygous for rad50s \\
\hline FNY40 . & ...FNY34 but homozygous for rad50s \\
\hline
\end{tabular}

The his4-100 and his4-108 alleles were constructed by ligating 30- and 38-bp palindromic oligonucleotides at the SalI site of pDN73, respectively. The his4100 allele has a PstI site, and the his4-108 allele has a XbaI site, at the center of symmetry. The his $4-105$ mutant allele was constructed by inserting a 35-bp-long palindromic oligonucleotide at the SalI site of pDN73. The resulting plasmid, pFN1, had a 105-bp palindromic insertion with a BstEII site at its center of symmetry. The his4-116 and his 4-120 alleles were constructed by adding 11- and 15-bp-long palindromic oligonucleotides at the BstEII site of pFN1, respectively. Both of these oligonucleotides have a BglII site at the center of symmetry. To generate the his4-140a allele, the plasmid pFN1 was digested with BstEII and ligated with another 35-bp palindromic oligonucleotide that had a BstEII linker at its end. The resulting plasmid, pFN2, had a 140-bp palindromic insertion. The his4-116, his4-120, and his4-140a alleles generate a single-base mismatch within the stem of the stem-loop structure at the site (BstEII) of the last insertion when present in hDNA. Since a mismatched base pair in the stem of the hairpin structure does not alter the repair ability of the yeast mismatch repair system (40), we used these mutant alleles for subsequent experiments. All constructions were confirmed by DNA sequencing. The unique SnaBI site within the HIS4 sequence, present in all of the constructs, was used for targeting.

The rad1 deletion plasmid pDG18 was kindly provided by Robert Schiestl (Harvard University, Cambridge, Mass.). The plasmid pDG18 had a 7-kb BamHI fragment containing a RAD1 sequence in which an internal HindIII fragment ( -212 to 3853 ) deleting most of the $R A D 1$ sequences was replaced with a $1-\mathrm{kb}$ HindIII fragment containing the URA3 gene (51).

Construction of $\mathbf{R e c}^{-}$mutants. The mei4 deletion-disruption mutant allele was introduced into the chromosome by a one-step gene replacement (OSR) procedure (49) using the NotI-XhoI-digested plasmid pTM6 (a gift from S. Roeder, Yale University, New Haven, Conn.; 34); the rad50 deletion mutant allele was introduced with EcoRI-digested pNKY83 (5). The spo11 deletion mutant was constructed using plasmid pGB518 (kindly provided by C. Giroux, Wayne State University, Detroit, Mich.), where the entire coding region was 
TABLE 2. Sequences of palindromic oligonucleotides used in this study ${ }^{a}$

Mutant allele

(insertion length [bp])
Sequence

his4-70 (70).........................5'
his 4-100 (100)......................5'
CCGAGTACTGTATGTGGATCAGGTAAGGTTGGGAGTCGACTCCCAACCTTACCTGATCCACATACAGTAC 3'
CCAACCTTACCTGATCCACATACAGTAC $3^{\prime}$

his4-105 (105).....................5' TCGAGTACTGTATGTGGATCAGGTAAGGTTGGGAGTCGAGCGTGTAATATCGGTCACCGATATTACACGCT CGACTCCCAACCTTACCTGATCCACATACAGTAC 3'

his4-108 (108).....................5' TCGAGTACTGTATGTGGATCAGGTAAGGTTGGGAGTCGAGTCGGATGAATGTCTCTAGAGACATTCATCCG ACTCGACTCCCAACCTTACCTGATCCACATACAGTAC 3'

his4-116 (116).....................5' TCGAGTACTGTATGTGGATCAGGTAAGGTTGGGAGTCGAGCGTGTAATATCGGTCACAGATCTGTCACCGA TATTACACGCTCGACTCCCAACCTTACCTGATCCACATACAGTAC 3'

his4-120 (120)......................' TCGAGTACTGTATGTGGATCAGGTAAGGTTGGGAGTCGAGCGTGTAATATCGGTCACTGAGATCTCAGTCA CCGATATTACACGCTCGACTCCCAACCTTACCTGATCCACATACAGTAC 3

his4-140a (140) ...................5' TCGAGTACTGTATGTGGATCAGGTAAGGTTGGGAGTCGAGCGTGTAATATCGGTCACTGCTAAAAGCGGA GATCTCCGCTTTTAGCAGTCACCGATATTACACGCTCGACTCCCAACCTTACCTGATCCACATACAGTAC 3'

\footnotetext{
${ }^{a}$ Each oligonucleotide contains a SalI-compatible 5' TCGA 3 ' sequence at the $5^{\prime}$ end. The restriction endonuclease site present at the center of symmetry of each oligonucleotide is in bold letters. The oligonucleotides were inserted at the SalI site within the HIS4 coding sequence as described in Materials and Methods.
}

replaced with the hisG-URA3-hisG cassette. The plasmid was digested with $X b a \mathrm{I}$ and $B g l \mathrm{II}$ before transformation (OSR). The rec114-2R, rec102- $\Delta 1:: U R A 3$, and rec104- $\Delta 1$ deletion mutants were obtained with plasmids pDP3.1, pCM211, and pAMG404- $\Delta 1$ (kindly provided by R. Malone, University of Iowa, Ames), respectively. The rec102 mutation was introduced by OSR with KpnI-digested pCM211, and the rec114 and rec104 mutations were introduced by a two-step gene replacement procedure with SnaBI-digested pDP3.1 and ClaI-digested pAMG404- $\Delta 1$, respectively. The xrs 2 and mre11 mutant alleles were introduced into the chromosome by OSR using plasmids pEI40 and pKJ112-5 (kindly provided by J. Haber, Brandeis University, Waltham, Mass.). The plasmid pEI40 (19) was digested with Bam HI and HindIII, and pKJ112-5 (1) was digested with Bam HI before transformation. The mer $: \because U R A 3$ disruption mutant allele was constructed as follows. An EcoRI-to-BamHI fragment containing the MER2 gene was cloned into EcoRI- and BamHI-digested pBluescript $\mathrm{SK}^{+}$(Stratagene, La Jolla, Calif.). This plasmid was kindly provided by S. Roeder. A 1-kb BamHI fragment containing the $U R A 3$ gene was inserted into the $B g l I I$ site within the $M E R 2$ coding sequence. The resulting plasmid, pCJ2, was digested with EcoRI and Bam $\mathrm{HI}$ before transformation.

Genetic techniques. Tetrad dissection and analysis were carried out as described before (40). All diploid strains were sporulated on plates at $25^{\circ} \mathrm{C}$, and tetrads were dissected on YPD plates. After 3 days of growth at $30^{\circ} \mathrm{C}$, the spore colonies were replica plated onto minimal medium to follow the segregation of heterozygous markers. The segregation patterns were scored after $24 \mathrm{~h}$ of growth at $30^{\circ} \mathrm{C}$. To follow the segregation of his 4 alleles derived from FNY34 and FNY37, standard allelism tests were carried out as described in reference 13 . In brief, the replicas of spore colonies were mated with tester strains that had either the his $4-140$ or the his $4-140$ a mutant allele. The mated colonies were then replica plated onto complete synthetic medium-adenine plates to select diploids. After overnight growth at $30^{\circ} \mathrm{C}$, the diploid cells were replica plated again onto complete synthetic medium-histidine plates and treated with UV light to increase mitotic recombination. $\mathrm{His}^{+}$papillations indicating the presence of the heteroalleles were scored after 2 days of growth at $30^{\circ} \mathrm{C}$.

Physical analysis of DSB formation. Diploid strains were sporulated in $1 \%$ potassium acetate as described by Nag and Kurst (40), and samples were collected at different times after induction of sporulation. For physical analysis, meiotic DNA was digested with $P v u I I$ and the resulting fragments were separated on a $0.8 \%$ agarose gel. The DNA was transferred to a nylon membrane that was then hybridized with an XhoI-BglII fragment of HIS4 as a probe. The probe was obtained from pDN42 (40) as an XhoI-XbaI fragment. For monitoring of DSB formation at the $A R G 4$ locus, a PstI fragment containing a portion of the $A R G 4$ coding region and its upstream sequences was used as a probe. Amounts of DSBs were quantified from the 24-h time point using a Molecular Dynamics PhosphorImager.

Estimation of recombination frequencies in different $\mathrm{Rec}^{-}$mutants. Homozygous spo13 $\mathrm{Rec}^{-}$diploid cells containing his4 and leu2 heteroalleles were streaked onto YPD plates, and after 2 days of growth at $30^{\circ} \mathrm{C}$, a single colony was inoculated into $3 \mathrm{ml}$ of YPD broth. After overnight growth at $30^{\circ} \mathrm{C}$, the culture was diluted 1,000 -fold with presporulation medium and allowed to grow at $30^{\circ} \mathrm{C}$ until the cell concentration reached $2 \times 10^{7}$ to $4 \times 10^{7} / \mathrm{ml}$. Cells were washed with $1 \%$ potassium acetate and suspended in $1 \%$ potassium acetate containing one-fifth of the nutritional requirements at a final concentration of $1.5 \times 10^{7}$ to
$2 \times 10^{7} / \mathrm{ml}$ in a total volume of $50 \mathrm{ml}$. Aliquots were taken out at different time intervals and plated on complete synthetic medium, on medium lacking histidine, and in medium lacking leucine after desired dilutions. After 3 to 4 days of growth at $30^{\circ} \mathrm{C}$, colonies were counted.

\section{RESULTS}

Palindrome-induced DSB formation requires genes involved in the formation of normal meiosis-specific DSBs. Nag and Kurst previously reported that the his4-140 mutant allele, generated by inserting a 140-bp inverted repeat within the HIS4 coding sequence, induces DSBs during meiosis (40). In the HIS4/his4-140 heterozygous strain, DSBs formed on the mutant chromosome at the palindromic-insertion site are repaired by homologous recombination using the wild-type chromosome as a template. It has been suggested that the DSBs at the palindromic-insertion site are due to extrusion of the palindromic sequences into cruciform conformation and subsequent cleavage by structure-specific nucleases. According to this hypothesis, DSBs at the palindromic-insertion site should be independent of the enzymes that make DSBs at the normal meiosis-specific DSB sites. Meiotic DSB formation requires the products of at least nine genes (22). Six of the nine genes are meiosis specific, i.e., SPO11, MEI4, MER2, REC102, $R E C 104$, and REC114. Spo11, a putative topoisomerase II-like transesterase that remains attached at the $5^{\prime}$ end of the DSB site, is believed to catalyze DSB formation $(8,22)$. However, it is yet to be proven that Spo11 actually makes the DSBs. The remaining three genes ( $R A D 50, M R E 11$, and $X R S 2)$ are also necessary for repair of DSBs in mitotic cells. A null mutation in any of these genes inhibits meiosis-specific DSB formation and thus decreases the meiotic level of recombination.

To investigate whether the DSB formation at the palindromic-insertion site occurs by a different mechanism, long-inverted-repeat-mediated meiotic recombination was followed in a strain that fails to form normal meiosis-specific DSBs. Since the biochemical role of each of these early recombination genes is not known, we constructed strains that are defective in each of these early recombination genes (Table 1). The diploid strain used in our studies is heteroallelic for his4-140 and his4-713 (a single-base-pair insertion) mutations present at the $5^{\prime}$ and $3^{\prime}$ end of the HIS4 gene, respectively. Since recombi- 
TABLE 3. Frequencies of meiotic recombination between his4 and leu2 heteroalleles in different early $\operatorname{Rec}^{-}$mutants $^{a}$

\begin{tabular}{|c|c|c|c|c|c|c|}
\hline \multirow{3}{*}{ Strain (allele) } & \multicolumn{6}{|c|}{ Recombination frequency at: } \\
\hline & \multicolumn{3}{|c|}{ his4 } & \multicolumn{3}{|c|}{ leu2 } \\
\hline & $0 \mathrm{~h}$ & $24 \mathrm{~h}$ & Fold increase & $0 \mathrm{~h}$ & $24 \mathrm{~h}$ & Fold increase \\
\hline CJY1 (wild type) & $9.3 \times 10^{-4}$ & $9.6 \times 10^{-2}$ & 103.2 & $2.0 \times 10^{-5}$ & $5.7 \times 10^{-3}$ & 285 \\
\hline CJY2 (mei4) & $7.9 \times 10^{-4}$ & $9.0 \times 10^{-4}$ & 1.1 & $1.6 \times 10^{-5}$ & $2.0 \times 10^{-5}$ & 1.3 \\
\hline CJY11 $(\operatorname{rad50})$ & $5.9 \times 10^{-4}$ & $8.5 \times 10^{-4}$ & 1.4 & $3.7 \times 10^{-4}$ & $3.1 \times 10^{-4}$ & 0.8 \\
\hline CJY15 (rec102) & $1.2 \times 10^{-3}$ & $1.1 \times 10^{-3}$ & 0.9 & $1.4 \times 10^{-5}$ & $1.0 \times 10^{-5}$ & 0.7 \\
\hline CJY19 (rec104) & $7.7 \times 10^{-4}$ & $8.4 \times 10^{-4}$ & 1.1 & $1.7 \times 10^{-5}$ & $1.7 \times 10^{-5}$ & 1 \\
\hline CJY30 (rec114) & $2.2 \times 10^{-3}$ & $3.3 \times 10^{-3}$ & 1.5 & $1.7 \times 10^{-5}$ & $2.2 \times 10^{-5}$ & 1.3 \\
\hline CJY25 (xrs2) & $5.3 \times 10^{-4}$ & $5.8 \times 10^{-4}$ & 1.1 & $\mathrm{ND}^{b}$ & ND & \\
\hline CJY24 (mre11) & $1.4 \times 10^{-3}$ & $3.7 \times 10^{-3}$ & 2.6 & $4.0 \times 10^{-4}$ & $8.8 \times 10^{-4}$ & 2.2 \\
\hline CJY57 (mer2) & $1.0 \times 10^{-3}$ & $9.0 \times 10^{-4}$ & 0.9 & $2.1 \times 10^{-5}$ & $1.3 \times 10^{-5}$ & 0.6 \\
\hline CJY29 (spo11) & $6.4 \times 10^{-4}$ & $9.6 \times 10^{-4}$ & 1.5 & $3.6 \times 10^{-5}$ & $4.0 \times 10^{-5}$ & 1.1 \\
\hline
\end{tabular}

${ }^{a}$ Sporulation was carried out as described in Materials and Methods. The data presented are averages of three independent experiments for each mutant strain. ${ }^{b} \mathrm{ND}$, not determined.

nation is necessary for proper segregation of chromosomes during the first division of meiosis, all early recombinationdefective $\left(\mathrm{Rec}^{-}\right)$mutants produce inviable spores (46). Spore viability can be rescued by introducing the spo13 mutation, which bypasses the first meiotic division (46). We introduced the spo13 mutation into our strain background to monitor recombination in a return-to-growth experiment.

As an internal control, we also used leu2 heteroalleles (leu2$B s t$ and leu2-RI). These two leu2 mutations are due to nonpalindromic insertions and are present near the $5^{\prime}$ and $3^{\prime}$ ends of the gene, respectively. Diploid cells with a mutation in any of the nine early recombination genes are incapable of forming normal meiosis-specific DSBs and thus fail to induce meiotic levels of recombination. Most of the meiotic recombination at the HIS4 locus occurs due to the DSB formation within the HIS4 promoter region. In our system, meiotic recombination at the HIS4 locus can occur only if the DSBs at the palindromic-insertion site occur by a mechanism that is different from that responsible for making DSBs at the HIS4 promoter. In such a case, meiotic recombination at the HIS4 locus is expected to be induced in $\mathrm{Rec}^{-}$mutants whereas the recombination frequency at the $L E U 2$ locus will remain at the mitotic level. Results of meiotic recombination with his 4 and leu2 heteroalleles are shown in Table 3.

In the recombination-proficient strain, meiotic recombination was induced over 100-fold at both the HIS4 and LEU2 loci, whereas in the $\mathrm{Rec}^{-}$background, the recombination frequency at both the HIS4 and LEU2 loci remained at the mitotic level (Table 3). These results suggest that DSBs at the palindromic-insertion site are formed by a mechanism that requires genes involved in normal meiosis-specific DSB formation. The DSB formation at the HIS4 locus was also monitored physically in the spol1 background, and the results are shown in Fig. 1B. DSBs both at the promoter region and at the palindrome were not observed in the spo11 background, suggesting that the normal recombination machinery is necessary for DSB formation at the palindrome.

The high rate of gene conversion events by long inverted repeats is independent of the primary sequence of the palindromic insertions. Since in previous studies by Nag and Kurst (40), only one long palindromic insertion was tested, there remains a possibility that the primary sequence of the inserted oligonucleotide has a role in DSB formation, rather than the palindromic nature of the inserted sequence. To demonstrate that it is the repeated sequence rather than the primary sequence of the inverted repeat that leads to DSB formation, we inserted a different 140-bp-long inverted repeat (Table 2) at the same SalI site that was used to construct the his4-140 mutant allele to generate the his4-140a mutant allele. A HIS4/ his4-140a heterozygous-diploid strain was sporulated, and the tetrads were analyzed. About $25 \%$ of the total number of unselected tetrads had aberrant events, and none of these were
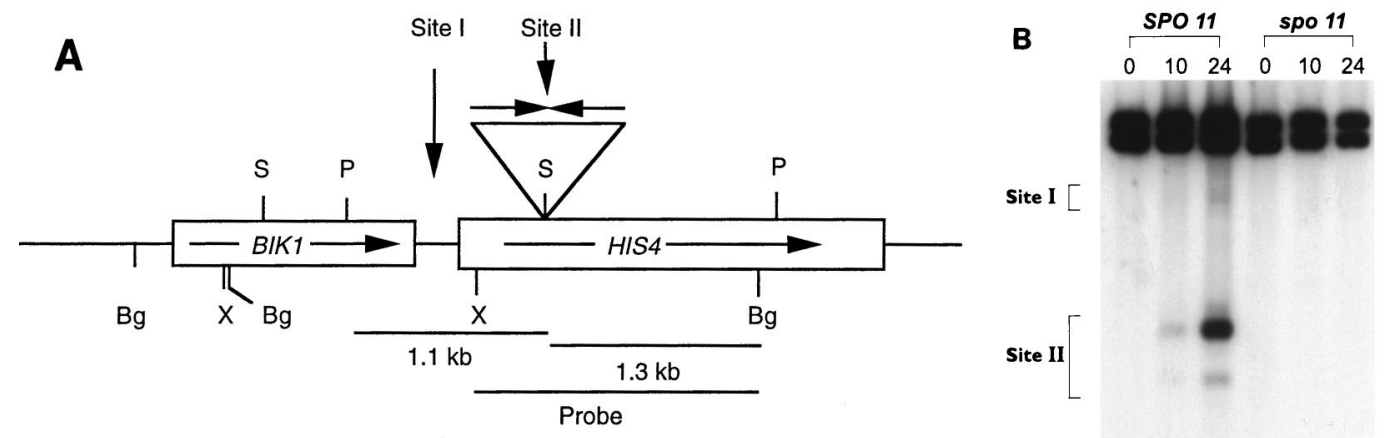

FIG. 1. DSB formation in the wild type and in cells homozygous for the spo11 mutation. (A) Partial restriction map of the HIS4-BIK1 region. Only relevant restriction sites are shown. The boxes indicate the coding regions of genes, and the arrows indicate the direction of transcription. The expanded region over the linear map represents palindromic insertions. Abbreviations: Bg, BglII; P, PvuII; S, SalI; X, XhoI. Site I represents the DSB site at the HIS4 promoter, and Site II represents the DSB site at the palindromic-insertion site. (B) Analysis of DSB formation. DNA was isolated from cells collected at various times (hours) after induction of meiosis and digested with PvuII before being run on a gel. The XhoI-BglII fragment containing most of the HIS4 coding region was used as a probe. The number above each lane indicates the time (in hours) of sample collection. A PvuII digestion of the meiotic DNA generates a 2,428-bp fragment for the HIS4/HIS4 diploid. 
TABLE 4. Segregation patterns of different his4 mutant alleles ${ }^{a}$

\begin{tabular}{|c|c|c|c|c|c|c|c|c|c|c|c|c|c|}
\hline Strain & Mutant locus & $6: 2$ & $2: 6$ & $8: 0$ & $0: 8$ & $5: 3$ & $3: 5$ & $\begin{array}{c}\text { Aberrant } \\
4: 4\end{array}$ & $\begin{array}{c}\text { Other } \\
\text { aberrants }^{b}\end{array}$ & $\begin{array}{l}\text { Total no. } \\
\text { of tetrads }\end{array}$ & $\begin{array}{l}\text { Aberrant } \\
\text { segregation } \\
(\% \text { of total })\end{array}$ & $\begin{array}{l}\text { Gene conversion } \\
\text { (\% of aberrants) }\end{array}$ & $\begin{array}{c}\text { PMS (\% of } \\
\text { aberrants) }\end{array}$ \\
\hline DNY64 ${ }^{c}$ & HIS4/his4-140 & 68 & 4 & 35 & 0 & 0 & 0 & 0 & 0 & 390 & 27.4 & 100 & 0 \\
\hline FNY4 & HIS4/his4-140a & 76 & 3 & 9 & 0 & 0 & 0 & 0 & 0 & 354 & 24.9 & 100 & 0 \\
\hline FNY10 & HIS4/his4-120 & 42 & 5 & 1 & 0 & 2 & 2 & 0 & 0 & 297 & 17.5 & 92.3 & 7.7 \\
\hline FNY18 & HIS4/his4-116 & 46 & 5 & 1 & 0 & 5 & 5 & 1 & 0 & 312 & 20.2 & 82.5 & 17.5 \\
\hline FNY6 & HIS4/his4-108 & 48 & 11 & 3 & 1 & 3 & 7 & 0 & 0 & 346 & 21.1 & 86.3 & 13.7 \\
\hline FNY2 & HIS4/his4-105 & 33 & 14 & 0 & 0 & 16 & 9 & 1 & 0 & 335 & 21.8 & 64.4 & 35.6 \\
\hline DNY225 & HIS4/his4-100 & 20 & 4 & 1 & 0 & 19 & 12 & 3 & 2 & 304 & 20.1 & 41.0 & 59.0 \\
\hline FNY16 & HIS4/his4-70 & 9 & 7 & 0 & 0 & 27 & 31 & 3 & 2 & 251 & 31.5 & 20.3 & 79.7 \\
\hline
\end{tabular}

${ }^{a}$ Diploids were sporulated at $25^{\circ} \mathrm{C}$ for 2 to 4 days, and the tetrads were dissected and analyzed as described in Materials and Methods. The frequencies of gene conversion for other markers $(2,199$ tetrads were analyzed) were $7.1 \%$ (arg4-17), $0.8 \%(\operatorname{trp} 1-1), 3.4 \%($ leu2-Bst $)$, and $3.2 \%($ tyr $7-1)$.

${ }^{b}$ Other classes include DNY225 (one aberrant 6:2 and one 1:7) and FNY16 (one aberrant 6:2 and one 1:7).

${ }^{c}$ Data for DNY64 are from reference 40.

PMS events (Table 4). In addition, there is a strong disparity between $6: 2$ and 2:6 events (76 versus 3 ) and the $6: 2$ and 8:0 events constituted $96 \%$ of the aberrants. This segregation pattern is characteristic of a meiotic recombination initiation site on the mutant chromosome. These results suggest that the his4-140a allele, like his4-140, acts as a meiotic recombination hot spot, or the gene conversion events associated with his4$140 a$ are generated by a mechanism that is independent of DSB formation at the palindromic-insertion site.

The above observation also raises the possibility that the length of the palindromic insertion is responsible for the high rate of gene conversion events since short palindromes (36 bp or less) produced mostly PMS events (40-42). This possibility is also consistent with the observation that short inverted repeats are highly stable in both prokaryotes and eukaryotes, and long inverted repeats are difficult to maintain in vivo, as they undergo severe rearrangements.

To understand the role of the length of the repeated units in palindrome-induced meiotic recombination, we constructed several his 4 mutant alleles by inserting inverted repeats of different lengths (Table 2) and the results of the tetrad analysis are shown in Table 4. A 70-bp palindromic-insertion mutant allele (his4-70), like short palindromic-insertion mutant alleles, exhibited a high rate of PMS events. About $80 \%$ of the total aberrants were PMS events with no significant disparity between $5: 3$ and $3: 5$ or between $6: 2$ and 2:6 events. However, as the length of the palindromic insertion was increased to $100 \mathrm{bp}$ or more, the rate of PMS events was decreased with a simultaneous increase in the rate of gene conversion events (Table 4 and Fig. 2). The his4 mutant alleles containing 108- and 120-bp inverted repeats had about 14 and 8\% PMS events, respectively, among the aberrants. In addition, the disparity between $6: 2$ and 2:6 events was higher with increased length of the repeated units. These results indicate that the length of the palindromic insertions is responsible for generating a high rate of conversion events and the disparity between 6:2 and 2:6 events.

Long-palindromic-insertion mutant alleles induce DSBs during meiosis. Since long-palindromic-insertion mutant alleles exhibit a segregation pattern that is similar to that of the his4-140 allele (Table 4), it is likely that all long inverted repeats induce DSBs during meiosis. To see whether the his4140 a mutant allele generates DSBs during meiosis, meiotic DNA was prepared from HIS4/his4-140a diploid cells (FNY8), digested with PvuII, and compared with that of the HIS4/HIS4 diploid strain (Fig. 3A). Both of these strains were homozygous for the rad50S mutation that prevents processing of DSBs (3).

PvuII digestion of DNY115 (HIS4/HIS4 rad50S/rad50S)
DNA generates a 2.4-kb fragment that contains most of the HIS4 gene and a portion of the BIK1 gene (Fig. 1A). Similar digestion of FNY8 (HIS4/his4-140a rad50S/rad50S) DNA would produce a $2.4-\mathrm{kb}$ fragment (resulting from the wildtype chromosome) and an $\sim 2.6$-kb fragment (resulting from the mutant chromosome). The results are shown in Fig. 3A. In addition to the parental DNA fragments, meiotic DNAs derived from both DNY115 and FNY8 exhibited bands characteristic of DSBs at the HIS4 promoter (site I). Most of the meiotic recombination at the HIS4 locus occurs due to DSBs at the HIS4 promoter region $(12,41)$. DNY115 DNA had one band, while FNY8 DNA had two bands separated by about 150 bp at site I representing promoter breaks on both the wild-type and mutant chromosomes.

In addition to the promoter breaks, the meiotic DNA from FNY8 showed the presence of a DNA fragment of about $1.4 \mathrm{~kb}$ that resulted from the DSB formation at the palindromicinsertion site (site II). The other expected $1.2-\mathrm{kb}$ fragment was not visible due to the short homology with the probe. Quantitative analyses have indicated that both DNY115 and FNY8 produced nearly equal percentages of breaks at the HIS4 promoter (3.3 to 4.6 and 3.4 to $4.3 \%$, respectively; Table 5). In a wild-type diploid strain, the percentage of DSBs at the promoter varies from 2 to $5 \%$ of the total meiotic DNA (12). The percentage of DSBs at the palindromic-insertion site (site II)

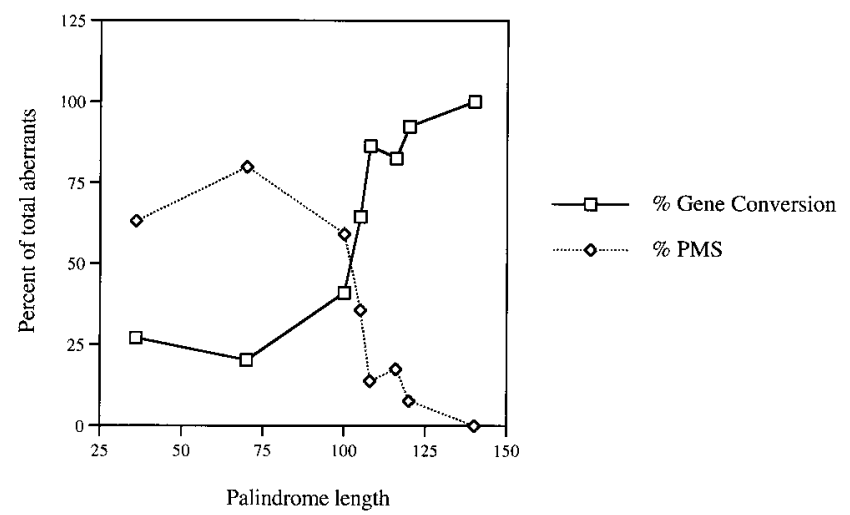

FIG. 2. Rates of gene conversion and PMS events with his4 mutant alleles containing palindromic insertions of different lengths. PMS and gene conversion events are shown as percentages of the total number of aberrant events. This graph is based on information provided in Table 4. The PMS and gene conversion rates for the 36-bp palindromic-insertion mutant allele (his4-9) are from reference 40 . 


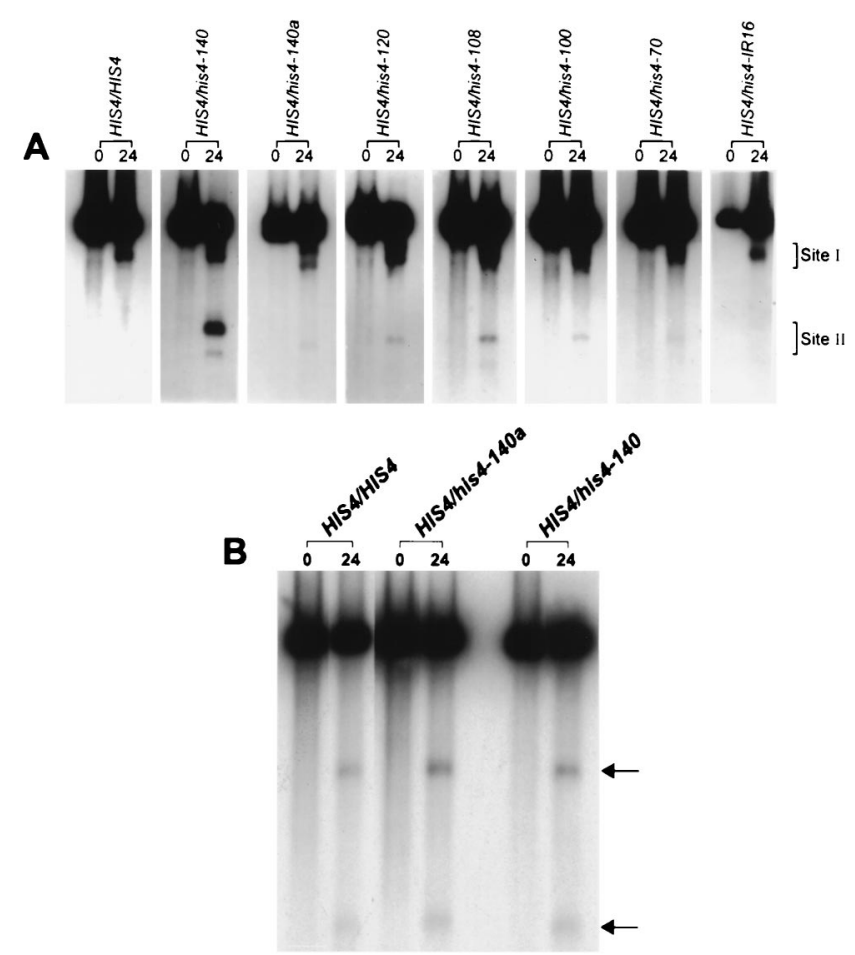

FIG. 3. DSB formation in the wild type and strains containing palindromic insertions of different lengths. (A) Analysis of DSB formation at the HIS4 locus. DNA was isolated at different times (hours) from cells undergoing meiosis. DSB analysis was carried out as described in the legend to Fig. 1. Site I represents DSBs at the HIS4 promoter, and site II represents DSBs at the palindromicinsertion site. The number above each lane indicates the time (hours) of sample collection. (B) DSB analysis at the ARG4 locus. DNA samples were the same ones that were used to analyze DSBs at the HIS4 locus. The numbers above the lanes indicate the times of sample collection. The arrows indicate fragments generated due to DSBs.

in FNY8 was 0.4 to $0.8 \%$ of the total meiotic DNA. This percentage is nearly 10 -fold lower than that produced by the his4-140 mutant allele (Fig. 3A and Table 5). This result suggests that although long palindromic sequences induce DSBs during meiosis, the percentage of breakage is determined by the primary sequence of the inserted palindromic sequence.

The his 4 mutant alleles containing palindromic insertions of different lengths were also studied physically to monitor their ability to induce DSBs. Each palindromic insertion, from 70 to $140 \mathrm{bp}$ in length, resulted in DSB formation at the palindromic-insertion site, ranging from 0.2 to $0.8 \%$ of the total meiotic DNA. The percentages of DSBs at the HIS4 promoter remained similar with and without palindromic insertions (2 to $6 \%$; Table 5). Surprisingly, the 70-bp palindromic insertion also had about 0.1 to $0.2 \%$ of its DNA as DSBs at site II even though the his4-70 mutant allele exhibited a high rate of PMS events. These results prompted us to investigate DSB formation with the 32-bp palindromic-insertion mutant allele (his4IR16). The inverted repeat that was used to generate the his4IR16 allele (41) is present in all of the palindromic sequences used in these experiments and in his4-140 (see Materials and Methods). As shown in Fig. 3A, the 32-bp insertion did not generate DSBs at a detectable level $(<0.1 \%)$. Our physical analysis also showed that a 36-bp palindromic-insertion mutant allele failed to induce DSBs at a detectable level (data not shown). The physical analyses with palindromic-insertion mutant alleles of different lengths suggest that inverted repeats longer than $50 \mathrm{bp}$ are likely to induce DSBs during meiosis, and the DSB amount depends on both the length of the repeated units and the primary sequences of the inverted repeats.

It should be noted that the small percentage of DSBs generated by his4-140a and other smaller-palindromic-insertion mutant alleles was not due to alterations in the strain background, since the recombination rates at the $A R G 4$ locus remained similar in DNY64 (7.4\%) and FNY4 (7.1\%). The DSB formation at the $A R G 4$ locus was also analyzed physically in DNY115 (HIS4/HIS4), DNY214 (HIS4/his4-140), and FNY8 (HIS4/his4-140a). These diploids are homozygous for the rad50S mutation. We used the same DNA preparations that were used to monitor DSBs at the HIS4 locus. The results are shown in Fig. 3B. The percentages of DSBs (data not shown) in these three strains remained similar, suggesting that the low level of DSB formation by different palindromic-insertion mutant alleles was not due to a global reduction of meiotic levels of DSB formation.

Gene conversion events by long-palindromic-insertion mutant alleles are generated by an MSH2- and RAD1-independent pathway. The physical analysis of different palindromicinsertion mutant alleles indicated that about $90 \%$ of the breaks were at the HIS 4 promoter and nearly $10 \%$ of the total breaks were at the palindromic-insertion site. This suggests that most of the gene conversion events were due to a recombination initiation event at the HIS4 promoter. However, DSBs at the HIS4 promoter would lead to hDNAs containing long hairpin structures. The results of tetrad analysis of diploid cells containing different palindromic-insertion mutant alleles suggest that hDNA containing long hairpin structures are preferentially repaired in favor of the wild-type strand, resulting in a disparity between 6:2 and 2:6 events. To investigate whether the repair of the hairpin structure occurs by an $M S H 2$-dependent pathway or by the $R A D 1$-dependent nucleotide excision repair pathway, we made $m s h 2$ derivatives of his $4-140 a$ and his 4-120 heterozygous diploid strains and a rad1 derivative of the his4-140a diploid strain. The Msh2-dependent mismatch repair system repairs mismatched base pairs and small loops $(25,26,36)$, and the Rad1-dependent nucleotide excision repair pathway repairs pyrimidine dimers and other helix distortion lesions (15). Recently, Rad1 has been shown to be involved in the repair of large loops in the hDNA that are formed during meiotic recombination in yeast (23). We were particularly interested in seeing the effect of the rad1 mutation in hairpin repair, since the hairpin structure might appear as a helix distortion to the mismatch repair system in yeast. The results of the tetrad analysis are shown in Table 6.

The presence of the msh 2 or radl mutation did not change the spectrum of events that were observed in the wild-type

TABLE 5. Percentages of DSBs produced by different his4-mutant alleles ${ }^{a}$

\begin{tabular}{lcc}
\hline \multirow{2}{*}{ HIS4 locus } & \multicolumn{2}{c}{ DSBs as \% of total meiotic DNA at: } \\
\cline { 2 - 3 } & HIS4 promoter & Palindrome \\
\hline HIS4/HIS4 & $3.3-4.6$ & 0 \\
HIS4/his4-140 & $2.8-3.8$ & $5.1-7.2$ \\
HIS4/his4-140a & $3.4-4.3$ & $0.4-0.8$ \\
HIS4/his4-120 & $4.9-6.4$ & $0.4-0.9$ \\
HIS4/his4-108 & $4.1-5.2$ & $0.2-0.6$ \\
HIS4/his4-100 & $3.2-4.3$ & $0.2-0.5$ \\
HIS4/his4-70 & $2.2-4.4$ & $0.1-0.2$ \\
\hline
\end{tabular}

${ }^{a}$ DSB formation was analyzed by Southern analysis of $P v u$ II-digested meiotic DNA, and percentages of DSBs were quantified from the 24-h time point. 


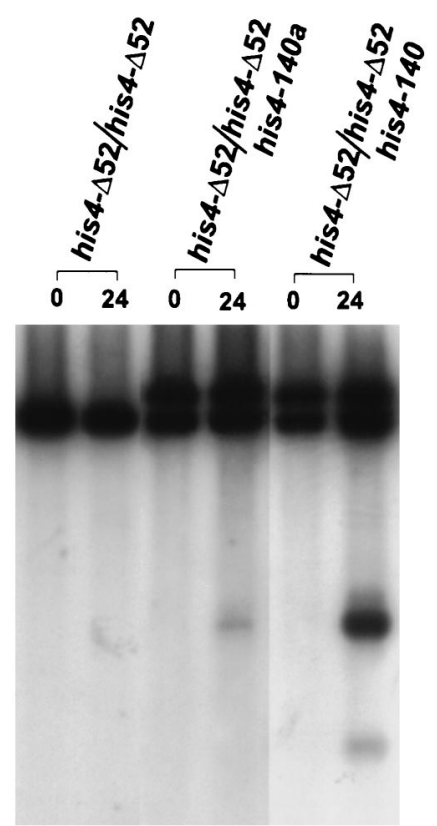

FIG. 4. Analysis of DSB formation in the HIS4 promoter deletion background. DNA was isolated from cells collected at different times after induction of meiosis, and physical analysis was carried out as described in the legend to Fig. 1. The numbers above the lanes indicate the times of sample collection.

background. In FNY31 (HIS4/his4-140a rad1/rad1), about 24\% of the unselected tetrads had aberrant events and none of the events were PMS events (Table 6). Similarly, the spectrum of events with his4-140a and his4-120 remained unaltered in the msh 2 background. These results suggest that the gene conversion events by long-palindromic-insertion mutant alleles were generated by repair of hDNA containing long hairpin structures by a pathway that is independent of the Msh2 and Rad1 enzymes. It is also possible that the gene conversion events were produced by a pathway not involving mismatch repair enzymes.

Deletion of the HIS4 promoter exhibits different effects on the recombination rate of different palindromic-insertion mutant alleles. The results presented above suggest that most of the gene conversion events associated with the his4-140a allele are due to DSBs at the HIS4 promoter hot spot. The above results also suggest that the disparity between $6: 2$ and 2:6 events at the his4-140a mutant allele was due to the preferential repair of the hairpin structure in the hDNA, whereas the same for his4-140 was due to repair of DSBs formed on the mutant chromosome at the palindromic-insertion site. According to this hypothesis, the deletion of the HIS4 promoter would reduce the recombination rate at the his $4-140 a$ allele while that at his 4-140 would remain nearly the same. To test this possibility, we constructed homozygous promoter deletion (his4$\Delta 52$ ) derivatives of HIS4/his4-140a (FNY37) and HIS4/his4140 (FNY34) diploid cells. These two diploid strains were sporulated, and the results of the tetrad analysis are shown in Table 7.

At the HIS 4 locus, about $60 \%$ of the meiotic recombination events are due to DSBs at the promoter region and the remaining events are due to recombination initiation outside the HIS4 promoter (24). The aberrant-segregation rate of the his4$140 a$ mutant allele in the promoter deletion background was reduced from 25 to $19 \%$, whereas that for his4-140 was increased from 27 to $33 \%$ (Table 7). The 8:0 events are likely to be due to double meiotic events. However, it is also possible that some of these events are due to DSBs that occurred before the premeiotic DNA replication. Considering that 8:0 events are due to double meiotic events, the aberrant-segregation rates are as follows: DNY64, 36.4\%; FNY34, 44.1\%; FNY4, $27.4 \%$; FNY37, 20.6\%. Using these values, the differences in the aberrant-segregation rates for his4-140a and his4-140 with and without the HIS4 promoter are significantly different $(P=$ 0.026 for his4-140 and $P=0.034$ for his4-140a; Fisher exact test). The disparity between 6:2 and 2:6 events (considering his $4-\Delta 52$ the wild-type allele and his $4-\Delta 52$ his $4-140$ or his $4-\Delta 52$ his $4-140 a$ the mutant allele) remained unaltered in both strains (Table 7). These results suggest that most of the events in HIS4/his4-140a diploid cells were due to recombination initiation at the HIS4 promoter and the HIS4 promoter hot spot slightly suppresses the other recombination initiation sites nearby.

We have also monitored the percentages of DSBs formed in the rad50S derivatives of FNY34 (FNY39) and FNY37 (FNY40) (Fig. 4). The percentages of DSBs at the palindrome at the his $4-140 a$ allele was increased from $\sim 0.6$ to $\sim 1.1 \%$ in the absence of the promoter, whereas the percentage of DSBs at his4-140 was increased from $\sim 6$ to $\sim 10.5 \%$ (Table 7). A similar difference in the amount of DSB formation was observed when the his4- $\Delta 52$ mutation and his4-140 were present in cis but not in trans (data not shown). These results suggest that two recombination initiation sites always compete with each other when present in cis.

\section{DISCUSSION}

Inverted repeats are a major source of genomic instability. This palindrome-mediated genomic instability is known to depend on the length of the inverted repeats; the longer the inverted repeat, the greater the possibility of rearrangements. Using both genetic and physical analyses, we have examined the effect of the presence of palindromic insertions of different lengths on meiotic recombination. Our results indicate that (i) long palindromic sequences induce DSBs during meiosis; (ii) palindrome-induced DSB formation requires the gene functions that are necessary for normal meiosis-specific DSB formation; (iii) the ability to induce DSBs depends on the lengths of palindromic insertions, and the percentages of DSBs depend on the primary sequence of the inverted repeats; and (iv) hDNAs containing long palindromic sequences are repaired or processed by a mechanism that does not involve Msh2 or Rad1 proteins, and the mismatches containing long hairpin struc-

TABLE 6. Segregation patterns of different his 4 mutant alleles in the rad1 and $m s h 2$ backgrounds

\begin{tabular}{|c|c|c|c|c|c|c|c|c|c|c|c|c|c|}
\hline Strain & Mutant locus & $6: 2$ & $2: 6$ & $8: 0$ & $0: 8$ & $5: 3$ & $3: 5$ & $\begin{array}{c}\text { Aberrant } \\
4: 4\end{array}$ & $\begin{array}{c}\text { Other } \\
\text { aberrants }\end{array}$ & $\begin{array}{l}\text { Total no. } \\
\text { of tetrads }\end{array}$ & $\begin{array}{c}\text { Aberrant } \\
\text { segregation } \\
(\% \text { of total) }\end{array}$ & $\begin{array}{l}\text { Gene conversion } \\
\text { (\% of aberrants) }\end{array}$ & $\begin{array}{c}\text { PMS (\% of } \\
\text { aberrants) }\end{array}$ \\
\hline DNY270 & HIS4/his4-120 msh2/msh2 & 32 & 10 & 0 & 1 & 3 & 2 & 0 & 0 & 224 & 21.4 & 90 & 10 \\
\hline FNY26 & HIS4/his4-140a msh2/msh2 & 20 & 1 & 1 & 0 & 0 & 0 & 0 & 0 & 139 & 15.8 & 100 & 0 \\
\hline FNY31 & HIS4/his4-140a rad1/rad1 & 49 & 8 & 5 & 0 & 0 & 0 & 0 & 0 & 261 & 23.8 & 100 & 0 \\
\hline
\end{tabular}


TABLE 7. Segregation patterns of different his4 mutant alleles in the promoter deletion background ${ }^{a}$

\begin{tabular}{|c|c|c|c|c|c|c|c|c|c|c|c|}
\hline \multirow{2}{*}{ Strain } & \multirow{2}{*}{ Mutant locus } & \multicolumn{4}{|c|}{$\begin{array}{c}\text { Gene } \\
\text { conversion }\end{array}$} & \multirow{2}{*}{ PMS } & \multirow{2}{*}{$\begin{array}{l}\text { Total no. } \\
\text { of tetrads }\end{array}$} & \multirow{2}{*}{$\begin{array}{l}\text { Aberrant } \\
\text { segregation } \\
\text { (\% of total) }\end{array}$} & \multirow{2}{*}{$\begin{array}{c}\text { Gene } \\
\text { conversion } \\
(\% \text { of aberrants) }\end{array}$} & \multicolumn{2}{|c|}{$\begin{array}{l}\text { Amt of DSBs as \% } \\
\text { of total DNA at: }\end{array}$} \\
\hline & & $6: 2$ & $2: 6$ & 8:0 & $0: 8$ & & & & & $\begin{array}{l}\text { Promoter } \\
\text { (site I) }\end{array}$ & $\begin{array}{c}\text { Insertion } \\
\text { (site II) }\end{array}$ \\
\hline DNY64 ${ }^{b}$ & HIS4/his4-140 & 68 & 4 & 35 & 0 & 0 & 390 & 27.4 & 100 & $2.8-3.8$ & $5.1-7.2$ \\
\hline FNY34 & his4- $\Delta 52 / h i s 4-\Delta 52$ his4-140 & 56 & 3 & 31 & 0 & 0 & 274 & 32.8 & 100 & & $9.8-11$ \\
\hline $\mathrm{FNY}^{b}{ }^{b}$ & HIS4/his4-140a & 76 & 3 & 9 & 0 & 0 & 354 & 24.9 & 100 & $3.4-4.3$ & $0.4-0.8$ \\
\hline FNY37 & his4- $\Delta 52 / h i s 4-\Delta 52$ his4-140a & 40 & 4 & 5 & 0 & 0 & 261 & 18.8 & 100 & & $1-1.3$ \\
\hline
\end{tabular}

${ }^{a}$ Sporulation and DSB analyses were carried out as described in Materials and Methods. Percentages of DSBs were quantified from 24-h samples and are percentages of the total meiotic DNA.

${ }^{b}$ Data are from Tables 4 and 5.

tures are preferentially repaired in favor of deletion of the hairpin structure.

DSB formation by the long-palindromic-insertion mutant allele (his4-140) at the HIS4 locus invoked the idea that long inverted repeats may undergo cruciform extrusion at a high frequency and such a structure may be processed by a structure-specific nuclease. One would then expect that the palindrome-induced DSB formation should be independent of enzymes or proteins that are responsible for making DSBs at normal meiosis-specific recombination initiation sites. As mentioned earlier, at least nine gene products are required to generate normal meiosis-specific DSBs (22). To determine whether these proteins are also necessary for DSB formation at the palindromic-insertion site, we monitored meiotic recombination between heteroalleles in all nine early $\mathrm{Rec}^{-}$mutants. In the $\mathrm{Rec}^{-}$background, meiotic levels of $\mathrm{His}^{+}$prototroph formation can occur if the DSBs at the palindrome occur by a different mechanism. It was necessary to study recombination initiation in all nine mutants. In the event that a particular mutant is not competent to make DSBs at the palindromicinsertion site, analysis of other early mutants may identify a mutant that is competent to make DSBs. This would indicate that the two mechanisms for DSB formation share some common proteins. However, our results have indicated otherwise. All nine genes are required for DSB formation at the palindromic-insertion site. The heteroallelic recombination frequency remained at the mitotic level in all nine mutants (Table 3 ), suggesting that DSBs at the palindrome and at the normal meiosis-specific sites occur by the same mechanism.

As mentioned earlier, normal meiosis-specific DSBs are believed to be Spo11 mediated. These meiosis-specific breaks normally occur at the nuclease-hypersensitive open chromatin structure. Although most loci exhibit similar hypersensitive sites during both meiosis and mitosis $(13,58,59)$, the DSB sites associated with $A R G 4$ and $C Y S 3$ are severalfold more hypersensitive during meiosis than during mitosis (43). It is possible that the presence of the palindromic sequences creates an open chromatin structure, which makes the sites available for the normal recombination machinery to make DSBs.

Although normal meiosis-specific DSB formation and palindrome-induced DSB formation require the same machinery, we cannot rule out the possibility of cruciform formation by the inserted inverted repeats, followed by cleavage of these stemloop structures by structure-specific nucleases. Recently, Jankowski et al. have shown that long CAG repeat tracts induce DSBs during meiosis in yeast and insertion of a nonrepeated DNA sequence at the same site fails to induce meiosisspecific DSBs (20). Both inverted repeats and CAG repeat tracts have the potential to form hairpin structures in vivo. It has also been shown that CAG repeats, like inverted repeats, when present in the single-stranded DNA, are likely to form hairpin structures in vivo (37). These results suggest that meiosis-specific DSB formation by the palindromic-insertion mutant alleles is related to the palindromic nature of the inserted sequence and also favors the hypothesis that DSB formation is due to cruciform extrusion by the inserted inverted repeats, followed by cleavage of the cruciform structure by structurespecific nuclease.

Palindrome-mediated genomic instability can be alleviated in $E$. coli by introducing $s b c C D$ mutations (27), and this protein complex possesses a hairpin-nicking activity (10). Two yeast proteins, Rad50 and Mre11, that are essential for meiotic recombination and DSB repair during mitosis share limited but significant homology with the $\mathrm{SbcC}$ and $\mathrm{SbcD}$ proteins, respectively (52). Recently, the human Mre11 protein, in addition to its $3^{\prime}$ to $5^{\prime}$ exonuclease activity, has been shown to cleave DNA hairpin loops (45). The yeast Mre11 protein has been shown to interact with Rad50 and Xrs2 (21). Yeast Mre11 possesses $3^{\prime}-5^{\prime}$ exonuclease and single-stranded endonuclease activities $(38,55,56)$. All of these activities are collectively required for the DSB-processing reaction and reside in the N-terminal half of Mre11. The C-terminal region of the protein is responsible for meiotic DSB formation and interacts with several meiosisspecific proteins $(38,56)$. It is possible that Mre11, in association with other meiotic proteins, including Spo11, forms a complex that is responsible for the cruciform cleavage activity. Such a hairpin-cleaving protein may require a long stem to make the DSB.

We also analyzed whether the primary sequence of the inserted inverted repeat in the his4-140 allele has any role in generating DSBs during meiosis by inserting a 140-bp-long inverted repeat of a different primary sequence (his4-140a mutant allele). The meiotic segregation pattern of his4-140a is similar to that of the his4-140 allele (Table 4). Only gene conversion events were observed with a strong disparity between 6:2 and 2:6 events. These results suggest that the his4$140 a$ mutant allele also acts as a hot spot for meiotic recombination and that the primary sequence of the inverted repeat may not have any role in inducing DSBs during meiosis. This conclusion was supported by the physical detection of DSBs in the HIS4/his4-140a diploid strain. However, the amount of DSBs produced by the his4-140a allele was 10-fold less than that produced by the his4-140 mutant allele (Table 5), suggesting that the sequence of the inserted oligonucleotide has some role in inducing meiosis-specific DSBs. The amount of DSB formation is determined not only by the primary sequence of the inverted repeat but also by the neighboring sequence. The insertion of the 140-bp inverted repeat, which created the his4-140 allele, at the LEU2 locus produced much less than $1 / 10$ of the DSBs made at the HIS4 locus (40). The insertion of the his4-140a inverted repeat created a new sequence environ- 
ment that may either lead to a lower level of cruciform formation or create a less accessible open chromatin structure.

Physical analyses involving the his4-140 and his4-140a mutant alleles indicate that the former acts as a stronger recombination initiation site than the latter. It has been shown that the presence of a strong recombination initiation site suppresses recombination initiation at a weak site nearby due to depletion of limiting factors $(13,58,59)$. In strains containing long-palindromic-insertion mutant alleles, two bands representing DSBs on wild-type and mutant chromosomes were observed at site I, and in DNY214 containing the his4-140 allele, one strong band (due to breaks on the wild-type chromosome) and a weak band (due to breaks on the mutant chromosome) were observed (Fig. 3A). The percentages of DSBs at the promoter on both the wild-type and mutant chromosomes were nearly the same when his4-140a was present, whereas his $4-140$ generated $2.3 \%$ and $1.0 \%$ DSBs on the wildtype and mutant chromosomes, respectively. These results indicate that there is competition between the recombination initiation sites when the his4-140 allele is present, whereas the his4-140a does not exert this effect. The his4-140a allele acts as a weak initiation site, and as a result, it does not have any effect on the promoter site. Tetrad analysis of his 4 promoter deletion mutants also indicated that a significant fraction of aberrant events originated due to promoter breaks when the his4-140a allele was present (Table 7).

Our tetrad analysis using his 4 mutant alleles containing palindromic insertions of different lengths showed that the rate of PMS events decreases with a simultaneous increase in the rate of gene conversion events as the length of the inverted repeats increases (Table 4 and Fig. 2). These results and physical analysis of DSB formation indicate that the ability to induce DSBs depends on the length of the inverted repeat. However, physical analysis showed that the amounts of DSBs produced by his 4 mutant alleles containing 100- to 140-bp insertions are nearly the same. Surprisingly, his4-70, a 70-bp palindromicinsertion mutant allele that exhibited a high rate of PMS events, also had about $0.2 \%$ of its total DNA as DSBs. About $1 / 10$ of the total number of breaks were at site II, and the rest were at site I, suggesting that most of the non-Mendelian events are due to recombination initiation at the HIS4 promoter.

Although the amount of breakage at the HIS4 promoter on the wild-type chromatid in the HIS4/his4-140a diploid strain remains the same as in HIS4/HIS4 diploid cells, the number of 2:6 events remains significantly low (Table 4). In addition, a significant level of DSBs is produced on the wild-type chromosome when the his4-140 allele is present (Fig. 3A). Again, the number of 2:6 events remains low. It is possible that because of large heterology, palindromic sequences are not included in the hDNA when the breaks are formed on the wild-type chromatid. However, this seems unlikely since larger heterologies are capable of being included in heteroduplexes (his4- $\Delta 52$, for example). Alternatively, some of the breaks at the promoter site may be repaired using the sister chromatid as a template.

The events initiated due to promoter breaks are expected to generate hDNA containing the inverted repeat as a long hairpin structure. The strong disparity between $6: 2$ and 2:6 events suggests that the hDNA containing the long hairpin structure is preferentially repaired in favor of the wild-type strand. As a result, when the wild type is the donor, the result is a 6:2 event and when the mutant is the donor, a 4:4 restoration event results. The Msh2-mediated repair pathway normally repairs mismatched base pairs and small loops $(25,26,36)$. The Rad1dependent nucleotide excision repair pathway is involved in repair of large loops that are formed in the hDNA during meiotic recombination (23). Introduction of a $m s h 2$ or $\mathrm{rad} 1$ mutation in our strain background did not change the spectrum of events that was observed in wild-type cells. The his4120 mutant allele produced 1.3 and $2.2 \%$ of total tetrads as PMS events in the wild type and in the msh 2 background, respectively. Similarly, the his4-140a allele had no PMS events in the wild type or in the msh 2 and radl backgrounds. These results suggest that there exists a repair pathway that is Msh2 and Rad1 independent and preferentially repairs the hairpin structure in favor of the wild-type strand. It is also possible that hDNAs containing long hairpin structures can be repaired by one of the two pathways: one is Msh2 dependent, and the other is Rad1 dependent. One of these two pathways is sufficient to correct the mismatch in the absence of the other.

The preferential repair of long hairpin structures raises another question: how do short hairpin structures escape repair? As proposed previously, proteins may exist that specifically bind at the hairpin structure. Such binding may occur at the base of the hairpin structure. When the hairpin structure is short, it may cover the entire stem-loop structure. In the case of a long hairpin structure, the binding of the protein at the base of the hairpin structure does not cover the entire stemloop structure, thereby exposing the loop region to the processing endonuclease, which then makes nicks in the loop of the hairpin structure. The nick then initiates the exonucleolytic degradation process to generate a gap, which is then repaired using the wild-type strand as a template. The same enzyme may be responsible for generating DSBs at the cruciform structure. Another possibility is that the formation of the nick depends on the length of the stem-loop structure, as the endonuclease may require a longer arm. Dependence on arm length has been observed with bacteriophage T4 endonuclease VII, which acts on Holliday junctions (39).

In summary, our results indicate that long inverted repeats serve as a major source of genomic instability by inducing DSBs during meiosis. Inverted-repeat-induced DSB formation depends on the length of the repeated units, and the amount of DSB formation is determined by the primary sequence of the inverted repeat. In addition, hDNAs containing long hairpin structures are repaired in favor of the deletion formation by a mechanism that does not require the normal cellular repair machinery.

\section{ACKNOWLEDGMENTS}

We thank the Molecular Genetics Core facility for providing oligonucleotides; Craig Giroux, Jim Haber, Robert Malone, Tom Petes, Shirleen Roeder, and Robert Schiestl for providing plasmids used in this study; and Andrew Reilly for his help with statistical analysis.

This work was supported by the NIH grant GM56266.

F.N. and C.J. contributed equally to this work.

\section{REFERENCES}

1. Ajimura, M., S.-H. Leem, and H. Ogawa. 1993. Identification of new genes required for meiotic recombination in Saccharomyces cerevisiae. Genetics 133:51-66.

2. Akgun, E., J. Zahn, S. Baumes, G. Brown, F. Liang, P. J. Romanienko, S. Lewis, and M. Jasin. 1997. Palindrome resolution and recombination in the mammalian germ line. Mol. Cell. Biol. 17:5559-5570.

3. Alani, E., R. Padmore, and N. Kleckner. 1990. Analysis of wild-type and rad50 mutants of yeast suggests an intimate relationship between meiotic chromosome synapsis and recombination. Cell 61:419-436.

4. Alani, E., R. A. G. Reenan, and R. D. Kolodner. 1994. Interaction between mismatch repair and genetic recombination in Saccharomyces cerevisiae. Genetics 137:19-39.

5. Alani, E., S. Suibbiah, and N. Kleckner. 1989. The yeast RAD50 gene encodes a predicted $153-\mathrm{kD}$ protein containing a purine nucleotide-binding domain and two large heptad repeat regions. Genetics 122:47-57.

6. Allers, T., and D. R. F. Leach. 1995. DNA palindromes adopt a methylationresistant conformation that is consistent with DNA cruciform or hairpin 
formation in vivo. J. Mol. Biol. 252:70-85.

7. Bennet, R. J., H. J. Dunderdale, and S. C. West. 1993. Resolution of Holliday junction by RuvC resolvase: cleavage specificity and DNA distortion. Cell 74:1021-1031

8. Bergerat, A., B. de Massy, D. Gadelle, P.-C. Varoutas, A. Nicolas, and P. Forterre. 1997. An atypical topoisomerase II from archaea with implications for meiotic recombination. Nature 386:414-417.

9. Collins, J. 1981. Instability of palindromic DNA in Escherichia coli. Cold Spring Harbor Symp. Quant. Biol. 45:409-416.

10. Connelly, J. C., L. A. Kirkham, and D. R. F. Leach. 1998. The SbcCD nuclease of Escherichia coli is a structural maintenance of chromosomes (SMC) family protein that cleaves hairpin DNA. Proc. Natl. Acad. Sci. USA 95:7969-7974.

11. Detloff, P. J., J. Sieber, and T. D. Petes. 1991. Repair of specific base pair mismatches formed during meiotic recombination in the yeast Saccharomyces cerevisiae. Mol. Cell. Biol. 11:737-745.

12. Fan, Q.-Q., F. Xu, and T. D. Petes. 1995. Meiosis-specific double-strand breaks at the HIS4 recombination hot spot in the yeast Saccharomyces cerevisiae: control in cis and trans. Mol. Cell. Biol. 15:1679-1688.

13. Fan, Q.-Q., F. Xu, M. White, and T. D. Petes. 1997. Competition between adjacent meiotic recombination hotspots in the yeast Saccharomyces cerevisiae. Genetics 145:661-670.

14. Fogel, S., R. K. Mortimer, and K. Lusnak. 1981. Mechanism of meiotic gene conversion, or "wanderings on a foreign strand," p. 289-339. In J. Strathern, E. W. Jones, and J. R. Broach (ed.), The molecular biology of the yeast Saccharomyces, vol. I. Cold Spring Harbor Laboratory, Cold Spring Harbor, N.Y.

15. Friedberg, E. C., G. C. Walker, and W. Siede. 1995. DNA repair and mutagenesis. American Society for Microbiology, Washington, D.C.

16. Glickman, B. W., and L. S. Ripley. 1984. Structural intermediates of deletion mutagenesis. Proc. Natl. Acad. Sci. USA 81:512-516.

17. Gordenin, D. A., K. S. Lobachev, N. P. Degtyareva, A. L. Malkova, E. Parkins, and M. A. Resnick. 1998. Inverted DNA repeats: a source of eukaryotic genomic instability. Mol. Cell. Biol. 13:5315-5322.

18. Hagen, N. F. P., S. D. Vincent, S. M. Ingleston, G. J. Sharples, R. J. Bennett, S. C. West, and R. G. Lloyd. 1998. Sequence-specificity of Holliday junction resolution: identification of RuvC mutants defective in metal binding and target site recognition. J. Mol. Biol. 281:17-29.

19. Ivanov, E. L., N. Sugawara, C. I. White, F. Fabre, and J. Haber. 1994. Mutations in XRS2 and RAD50 delay but do not prevent mating-type switching in Saccharomyces cerevisiae. Mol. Cell. Biol. 14:3414-3425.

20. Jankowski, C., F. Nasar, and D. K. Nag. 2000. Meiotic instability of CAG repeat tracts occurs by double-strand break repair in yeast. Proc. Natl. Acad. Sci. USA 97:2134-2139.

21. Johzuka, K., and H. Ogawa. 1995. Interaction of Mre11 and Rad50: two proteins required for DNA repair and meiosis-specific double-strand break formation in Saccharomyces cerevisiae. Genetics 139:1521-15332.

22. Keeney, S., C. N. Giroux, and N. Kleckner. 1997. Meiosis-specific DNA double-strand breaks are catalyzed by Spo11, a member of a widely conserved protein family. Cell 88:375-384.

23. Kirkpatrick, D. T., and T. D. Petes. 1997. Repair of DNA loops involves DNA-mismatch and nucleotide-excision repair proteins. Nature 387:929931.

24. Kirkpatrick, D. T., Y.-H. Wang, M. Dominska, J. D. Griffith, and T. D. Petes. 1999. Control of meiotic recombination and gene expression in yeast by a simple repetitive DNA sequence that excludes nucleosomes. Mol. Cell. Biol. 19:7661-7671.

25. Kolodner, R. 1996. Biochemistry and genetics of eukaryotic mismatch repair. Genes Dev. 10:1433-1442.

26. Kolodner, R., and G. T. Marsischky. 1999. Eukaryotic DNA mismatch repair. Curr. Opin. Genet. Dev. 9:89-96.

27. Leach, D. R. F. 1994. Long DNA palindromes, cruciform structures, genetic instability and secondary structure repair. Bioessays 16:893-900.

28. Leach, D. R. F., E. A. Okley, and D. J. Pinder. 1997. Repair by recombination of DNA containing a palindromic sequence. Mol. Microbiol. 26:597-606.

29. Lichten, M., and A. S. H. Goldman. 1995. Meiotic recombination hotspots. Annu. Rev. Genet. 29:423-444.

30. Lilley, D. M. J. 1980. The inverted repeat as a recognizable structural feature in supercoiled DNA molecules. Proc. Natl. Acad. Sci. USA 77:6468-6472.

31. Lobachev, K. S., B. M. Shor, H. T. Tran, W. Taylor, J. D. Keen, M. A. Resnick, and D. Gordenin. 1998. Factors affecting inverted repeat stimulation of recombination and deletion in Saccharomyces cerevisiae. Genetics 148: $1507-1524$

32. Maniatis, T., E. F. Fritsch, and J. Sambrook. 1983. Molecular cloning: a laboratory manual. Cold Spring Harbor Laboratory, Cold Spring Harbor, N.Y.

33. McClellan, J. A., P. Boublikova, E. Palecek, and D. Lilley. 1990. Superhelical torsion in cellular DNA responds directly to environmental and genetic factors. Proc. Natl. Acad. Sci. USA 87:8373-8377.
34. Menees, T. M., and G. S. Roeder. 1989. MEI4, a yeast gene required for meiotic recombination. Genetics 123:675-682.

35. Mizuuchi, K., M. Mizuuchi, and M. Gellert. 1982. Cruciform structures in palindromic DNA are favored by DNA supercoiling. J. Mol. Biol. 156:229253.

36. Modrich, P., and R. Lahue. 1996. Mismatch repair in replication fidelity, genetic recombination, and cancer biology. Annu. Rev. Biochem. 65:101133.

37. Moore, H., P. W. Greenwell, C.-P. Liu, N. Arnheim, and T. D. Petes. 1999. Triplet repeats form secondary structures that escape DNA repair in yeast. Proc. Natl. Acad. Sci. USA 96:1504-1509.

38. Moreau, S., J. R. Ferguson, and L. S. Symington. 1999. The nuclease activity of Mre11 is required for meiosis but not for mating type switching, end joining, or telomere maintenance. Mol. Cell. Biol. 19:556-566.

39. Mueller, J. E., C. J. Newton, F. Jensch, B. Kemper, R. Cunningham, et al. 1990. Resolution of Holliday junction analogs by T4 endonuclease VII can be directed by substrate structure. J. Biol. Chem. 265:13918-13924.

40. Nag, D. K., and A. Kurst. 1997. A 140-bp-long palindromic sequence induces double-strand breaks during meiosis in the yeast Saccharomyces cerevisiae. Genetics 146:835-847.

41. Nag, D. K., and T. D. Petes. 1993. Physical detection of heteroduplexes during meiotic recombination in the yeast Saccharomyces cerevisiae. Mol. Cell. Biol. 13:2324-2331.

42. Nag, D. K., M. A. White, and T. D. Petes. 1989. Palindromic sequences in heteroduplex DNA inhibit mismatch repair in yeast. Nature 340:318-320.

43. Ohta, K., T. Shibata, and A. Nicolas. 1994. Changes in chromatin structure at recombination initiation sites during yeast meiosis. EMBO J. 13:57545763

44. Paques, F., and J. E. Haber. 1999. Multiple pathways of recombination induced by double-strand breaks in Saccharomyces cerevisiae. Microbiol. Mol. Biol. Rev. 63:349-404.

45. Paull, T. T., and M. Gellert. 1998. The $3^{\prime}$ to $5^{\prime}$ exonuclease activity of Mre11 facilitates repair of DNA double-strand breaks. Mol. Cell 1:969-979.

46. Petes, T. D., R. E. Malone, and L. S. Symington. 1991. Recombination in yeast, p. 407-521. In J. Broach, J. R. Pringle, and E. W. Jones (ed.), The molecular and cellular biology of the yeast Saccharomyces, vol. I. Cold Spring Harbor Laboratory, Cold Spring Harbor, N.Y.

47. Rosche, W. A., T. Q. Trinh, and R. R. Sinden. 1995. Differential DNA secondary structure-mediated deletion mutation in the leading and lagging strands. J. Bacteriol. 177:4385-4391.

48. Rose, M. D., F. Winston, and P. Hieter. 1990. Methods in yeast genetics. Cold Spring Harbor Laboratory Press, Cold Spring Harbor, N.Y.

49. Rothstein, R. 1991. Targeting disruption, replacement, and allele rescue: integrative DNA transformation in yeast. Methods Enzymol. 19:281-301.

50. Ruskin, B., and G. R. Fink. 1993. Mutations in POL1 increase the mitotic instability of tandem inverted repeats in S. cerevisiae. Genetics 133:43-56.

51. Schiestl, R., J. Zhu, and T. D. Petes. 1994. Effect of mutations in genes affecting homologous recombination on restriction enzyme-mediated and illegitimate recombination in Saccharomyces cerevisiae. Mol. Cell. Biol. 14: 4493-4500.

52. Sharples, G. J., and D. R. Leach. 1995. Structural and functional similarities between the SbcCD proteins of Escherichia coli and the Rad50 and Mre11 (Rad32) recombination and repair proteins of yeast. Mol. Microbiol. 17: 1215-1217.

53. Sinden, R. R., G. Zheng, R. G. Brankamp, and K. N. Allen. 1991. On the deletion of inverted repeated DNA in Escherichia coli: effects of length, thermal stability and cruciform formation in vivo. Genetics 129:991-1005.

54. Trinh, T. Q., and R. R. Sinden. 1991. Preferential DNA secondary structure mutagenesis in the lagging strand of replication in E. coli. Nature 352:544 547.

55. Tsubouchi, T., and H. Ogawa. 1998. A novel mre11 mutation impairs processing of double-strand breaks of DNA during both mitosis and meiosis. Mol. Cell. Biol. 18:260-268.

56. Usui, T., T. Ohta, H. Oshiumi, J.-I. Tomizawa, H. Ogawa, and T. Ogawa. 1998. Complex formation and functional versatility of Mre11 of budding yeast in recombination. Cell 95:705-716.

57. Weston-Hafer, K., and D. E. Berg. 1991. Limits to the role of palindromy in deletion formation. J. Bacteriol. 173:315-318.

58. Wu, T.-C., and M. Lichten. 1995. Factors that affect the location and frequency of meiosis-induced double-strand breaks in Saccharomyces cerevisiae. Genetics 140:55-66.

59. Xu, L., and N. Kleckner. 1995. Sequence non-specific double-strand breaks and interhomolog interactions prior to double-strand break formation at a meiotic recombination hotspot in yeast. EMBO J. 14:5115-5128.

60. Zheng, G., T. J. Kochel, R. W. Hoepfner, S. E. Timmons, and R. R. Sinden. 1991. Torsionally tuned cruciform and Z-DNA probes for measuring unrestrained supercoiling at specific sites in DNA of living cells. J. Mol. Biol. 221:107-129. 